\title{
A Duality Transform for Constructing Small Grid Embeddings of 3D Polytopes ${ }^{\star}$
}

\author{
Alexander Igamberdiev and André Schulz \\ Institut für Mathematische Logik und Grundlagenforschung, \\ Universität Münster, Germany \\ $\{$ alex.igamberdiev, andre.schulz\}@uni-muenster.de
}

\begin{abstract}
We study the problem of how to obtain an integer realization of a $3 \mathrm{~d}$ polytope when an integer realization of its dual polytope is given. We focus on grid embeddings with small coordinates and develop novel techniques based on Colin de Verdière matrices and the MaxwellCremona lifting method.

As our main result we show that every truncated $3 \mathrm{~d}$ polytope with $n$ vertices can be realized on a grid of size polynomial in $n$. Moreover, for a class $\mathcal{C}$ of simplicial $3 \mathrm{~d}$ polytopes with bounded vertex degree, at least one vertex of degree 3 , and polynomial size grid embedding, the dual polytopes of $\mathcal{C}$ can be realized on a polynomial size grid as well.
\end{abstract}

\section{Introduction}

By Steinitz's theorem the graphs of convex 3d polytopes 1 are exactly the planar 3-connected graphs 16. Several methods are known for realizing a planar 3 -connected graph $G$ as a polytope with graph $G$ on the grid 471112 1315. It is challenging to find algorithms that produce polytopes with small integer coordinates. Having a realization with small grid size is a desirable feature, since then the polytope can be stored and processed efficiently. Moreover, grid embeddings imply good vertex and edge resolution. Hence, they produce "readable" drawings.

In $2 \mathrm{~d}$, it is well known that planar 3-connected graphs with $n$ vertices can be drawn on a $O(n) \times O(n)$ grid without crossings [5, and a drawing with convex faces can be realized on a $O\left(n^{3 / 2} \times n^{3 / 2}\right)$ grid [2]. For the realization as a polytope the best algorithm guarantees an integer embedding with coordinates at most $O\left(147.7^{n}\right)$ [311. The current best lower bound is $\Omega\left(n^{3 / 2}\right)$ [1. Closing this large gap is probably one of the most interesting open problems in lower dimensional polytope theory. Recently, progress has been made for a special class of $3 \mathrm{~d}$ polytopes, the so-called stacked polytopes. A stacking operation replaces a triangular face of a polytope with a tetrahedron, while maintaining the convexity of the embedding. A polytope that can be constructed from a tetrahedron and a

\footnotetext{
* This work was funded by the German Research Foundation (DFG) under grant SCHU 2458/2-1.

${ }^{1}$ In our terminology polytopes are always considered convex.
}

S. Wismath and A. Wolff (Eds.): GD 2013, LNCS 8242, pp. 173-184, 2013.

(C) Springer International Publishing Switzerland 2013 
sequence of stacking operation is called stacked polytope. The graphs of stacked polytopes are planar 3-trees. Stacked polytopes can be embedded on a grid that is polynomial in $n$ [6]. This is, however, the only nontrivial polytope class for which such an algorithm is known.

In this paper we introduce a duality transform that maintains a polynomial grid size. In other words, we provide a technique that takes a grid embedding of a polytope with graph $G$ and generates a grid embedding of a polytope whose skeleton is $G^{*}$, the dual graph of $G$. We call a 3 d polytope with graph $G^{*}$ a dual polytope. If the original polytope has integer coordinates bounded by a polynomial in $n$, then the dual polytope obtained with our techniques has also integer coordinates bounded by a (different) polynomial in $n$. Our methods can only be applied to special polytopes. Namely, we require that the graph of the polytope is a triangulation (the polytope is simplicial), that it contains a $K_{4}$, and that the maximum vertex degree is bounded.

For the class of stacked polytopes (although their maximum vertex degree is not bounded) we can also apply our approach to show that all graphs dual to planar 3-trees can be embedded as polytopes on a polynomial size grid. These polytopes are known as truncated polytopes. Truncated polytopes are simple polytope, that can be generated from a tetrahedron and a sequence of truncations. A truncation is the dual operation to stacking. This means that a degree-3 vertex of the polytope is cut off by adding a new bounding hyperplane that separates this vertex from the remaining vertices of the polytope. We show that all truncated polytopes can be realized with integer coordinates in $O\left(n^{44}\right)$. The approach for this class is more direct, since stronger results for realizations of stacked polytopes on the grid are known [6].

Duality. There exist several natural approaches how to construct for a given polytope a dual. The most prominent construction is polarity. Let $P$ be some polytope that contains the origin. Then $P^{*}=\left\{y \in \mathbb{R}^{d}: x^{T} y \leq 1\right.$ for all $\left.x \in P\right\}$ is a realization of a polytope dual to $P$, called its polar. The vertices of $P^{*}$ are intersection points of planes with integral normal vectors, and hence not necessarily integer points. In order to scale to integrality one has to multiply $P^{*}$ with the product of all denominators of its vertex coordinates, which may cause an exponential increase of the grid size.

A second approach uses the classic Maxwell-Cremona correspondence technique (also known as lifting approach) [10], which is applied in many embedding algorithms for $3 \mathrm{~d}$ polytope realization. The idea here is to first draw the graph of the polytope as a convex $2 \mathrm{~d}$ embedding with an additional equilibrium condition. The equilibrium condition guarantees that the $2 \mathrm{~d}$ drawing is a projection of a convex $3 \mathrm{~d}$ polytope, furthermore the polytope can be reconstructed from its projection in a canonical way (called lifting) in linear time. There is a classical transformation that constructs for a $2 \mathrm{~d}$ drawing in equilibrium a $2 \mathrm{~d}$ drawing of its dual graph, also in equilibrium. This drawing is called the reciprocal diagram. The induced lifting realizes the dual polytope, but it does not provide small integer coordinates for two reasons. First, the weights that define the equilibrium of the reciprocal diagram are the reciprocals of the weights in the original graph. 
Second, the lifting realizes the dual polytope in projective space with one point "over the horizon". The second property can be "fixed" with a projective transformation. This, however, makes a large scaling factor for an integer embedding unavoidable. Also the reciprocal weights are difficult to handle without scaling by a large factor.

Structure and notation. As a novelty we work with Colin de Verdière matrices to construct small grid embeddings. In order to make these techniques (as introduced by Lovász) applicable we extend this framework slightly; see Sect. 2. In Sect. 3 we then present the main idea, combining the classical lifting approach with the methods of Sect. 2, which finds applications in the following sections, where the results on truncated polytopes and triangulations are presented.

Throughout the paper we denote by $G$ the graph of the original polytope, and by $G^{*}$ its dual graph. For any graph $H$ we write $V(H)$ for its vertex set, $E(H)$ for its edge set and $N(v, H)$ for the set of neighbors of a vertex $v$ in $H$. Since we consider 3-connected planar graphs, the facial structure of the graph is predetermined up to a global reflection [17. The set of faces is therefore predetermined, and we name it $F(H)$. For convenience we denote an edge $\left(v_{i}, v_{j}\right)$ as $(i, j)$. A face spanned by vertices $v_{i}, v_{j}$, and $v_{k}$ is denoted as $\left(v_{i} v_{j} v_{k}\right)$. A graph obtained from $H$ by stacking a vertex $v_{1}$ on a face $\left(v_{2} v_{3} v_{4}\right)$, is denoted as $\operatorname{Stack}\left(H ; v_{1} ; v_{2} v_{3} v_{4}\right)$. For convenience we use $|p|$ for the Euclidean norm of the vector $p$. We denote the maximum vertex degree of a graph $G$ as $\Delta_{\mathrm{G}}$. Finally, we write $G[X]$ for the induced subgraph of a vertex set $X \subseteq V(G)$.

\section{3d Representations with CDV Matrices}

In this section we review some of the methods Lovász introduced in his paper on Steinitz representations [9]. In our constructions throughout the paper every face of any graph is realized such that all its vertices lie on a common plane. From this perspective drawings of graphs in $\mathbb{R}^{3}$ and the realizations of their corresponding polyhedra are the same objects.

Definition 1. We call a straight-line embedding $\left(u_{1}, \ldots, u_{n}\right) \in\left(\mathbb{R}^{3}\right)^{n}$ of a planar 3-connected graph $G$ in $\mathbb{R}^{3}$ a cone-convex embedding iff the cones over its faces, $C_{f}=\{\lambda x \mid x \in f, \lambda>0\}, f \in F(G)$ are convex and have disjoint interiors.

In other words, an embedding is a cone-convex embedding if its projection to the sphere $S=\{|x|=1\}$ is a convex drawing of $G$ with edges drawn as geodesic arcs. We remark that the vertices of a cone-convex embedding are not supposed to form a convex polytope.

Definition 2. Let $\left(u_{1}, \ldots, u_{n}\right)$ be an embedding of a graph $G$ into $\mathbb{R}^{d}$. We call a symmetric matrix $M=\left[M_{i j}\right]_{1 \leq i, j \leq n}$ a CDV matrix of the embedding if

1. $M_{i j}=0$ for $i \neq j,(i, j) \notin E(G)$, and

2. $\sum_{1 \leq j \leq n} M_{i j} u_{j}=0$ for $1 \leq i \leq n$.

We call a $C D V$ Matrix positive if $M_{i j}>0$ for all $(i, j) \in E(G)$. 
We call the second condition in the above definition the CDV equilibrium condition.

The CDV equilibrium condition can also be expressed in a slightly different, more geometric form as

$$
\sum_{j \in N(i, G)} M_{i j} u_{j}=-M_{i i} u_{i} \quad \text { for } 1 \leq i \leq n .
$$

Hence, a positive CDV Matrix witnesses that every vertex of the embedding can be written as a convex combination of its neighbors using symmetric weights. The following lemma appears in 9, we include the proof since it illustrates how to construct a realization out of a CDV matrix.

Lemma 1 (Lemma 4, [9]). Let $\left(u_{1}, \ldots, u_{n}\right)$ be a cone-convex embedding of a graph $G$ with a positive $C D V$ matrix $\left[M_{i j}\right]$. Then every face $f$ in $G$ can be assigned a vector $\phi_{f}$ s.t. for each adjacent face $g$ and separating edge $(i, j)$

$$
\phi_{f}-\phi_{g}=M_{i j}\left(u_{i} \times u_{j}\right),
$$

where $f$ lies to the left and $g$ lies to the right from $\overrightarrow{u_{i} u_{j}}$. The set of vectors $\left\{\phi_{f}\right\}$ is uniquely defined up to translations.

Proof. To construct the family of vectors $\left\{\phi_{f}\right\}$, we start by assigning an arbitrary value to $\phi_{f_{0}}$ (for an arbitrary face $f_{0}$ ); then we proceed iteratively. To prove the consistency of the construction, we show that the vectors $\left(\phi_{f}-\phi_{g}\right)$ sum to zero over every cycle in $G^{*}$. Since $G$ as well as $G^{*}$ is planar and 3-connected, it suffices to check this condition for all elementary cycles of $G^{*}$, which are the faces of $G^{*}$. Let $\tau(i)$ denote the set of counterclockwise oriented edges of the face in $G^{*}$ dual to $v_{i} \in V(G)$. Then, combining 1 and 2 yields

$\sum_{(f, g) \in \tau(i)}\left(\phi_{f}-\phi_{g}\right)=\sum_{j \in N(i, G)} M_{i j}\left(u_{i} \times u_{j}\right)=u_{i} \times\left(\sum_{j \in N(i, G)} M_{i j} u_{j}\right)=u_{i} \times\left(-M_{i i} u_{i}\right)=0$.

The vectors $\left\{\phi_{f}\right\}$ are unique up to the initial choice of $\phi_{f_{0}}$.

Note that there is a canonical way to derive a CDV matrix from a $3 \mathrm{~d}$ polytope 9]. Every $3 d$ embedding of a graph $G$ as a polytope $\left(u_{i}\right)$ possesses a positive CDV matrix defined by the vertices $\left(\phi_{i}\right)$ of its polar and equation (2). We refer to this matrix as the canonical $C D V$ matrix.

The following theorem, which is a variation of Lemma 5 in [9], is the main tool in our construction.

Theorem 1 (based on Lovász [9]). Let $\left(u_{1}, \ldots, u_{n}\right)$ be a cone-convex embedding of a graph $G$ and $M$ a positive $C D V$ matrix for this embedding. Then for any set of vectors $\left\{\phi_{f}\right\}_{f \in F(G)}$ fulfilling (2), the convex hull $\operatorname{Conv}\left(\left\{\phi_{f}\right\}_{f \in F(G)}\right)$ is a convex polytope with graph $G^{*}$; and the isomorphism between $G^{*}$ and the skeleton of $\operatorname{Conv}\left(\left\{\phi_{f}\right\}_{f \in F(G)}\right)$ is given by $f \rightarrow \phi_{f}$.

The proof of the theorem is included in the full version of the paper. It relies on a projection of the cone-convex embedding onto the sphere and an appropriate "scaling" of the CDV matrix. 


\section{Construction of Cone-Convex Embeddings}

In this section we describe how to go from a convex $2 \mathrm{~d}$ embedding with a positive equilibrium stress to a cone-convex $3 \mathrm{~d}$ embedding with a positive CDV matrix.

Definition 3. We call a set of reals $\left\{\omega_{i j}\right\}_{(i, j) \in E(G)}$ an equilibrium stress for an embedding $\left(u_{1}, \ldots, u_{n}\right)$ of a graph $G$ into $\mathbb{R}^{d}$ if for each $i \in V(G)$

$$
\sum_{j \in N(i, G)} \omega_{i j}\left(u_{j}-u_{i}\right)=0 .
$$

We call an equilibrium stress of a $2 d$ embedding with a distinguished boundary face $f_{0}$ positive if it is positive on every edge that does not belong to $f_{0}$.

The concept of equilibrium stresses plays a central role in the classical MaxwellCremona lifting approach and it is also a crucial concept in our embedding algorithm. The equilibrium stress on a realization of a complete graph arises as a "building block" in later constructions. The complete graph $K_{n}$, embedded in $\mathbb{R}^{n-2}$, has a unique equilibrium stress up to multiplication with a scalar. This stress has an easy expression in terms of volumes related to the embedding. We use the square bracket notation 2

$$
\left[q_{i} q_{j} q_{k} q_{l}\right]:=\operatorname{det}\left(\begin{array}{cccc}
x_{i} & x_{j} & x_{k} & x_{l} \\
y_{i} & y_{j} & y_{k} & y_{l} \\
z_{i} & z_{j} & z_{k} & z_{l} \\
1 & 1 & 1 & 1
\end{array}\right), \quad \text { where } q=\left(\begin{array}{c}
x \\
y \\
z
\end{array}\right)
$$

to obtain a formulation for the equilibrium stress on the $K_{5}$ embedding.

Lemma 2 (Rote, Santos, and Streinu [14]). Let $\left(u_{0}, u_{1}, \ldots, u_{4}\right)$ be an integer embedding of the complete graph $K_{5}$ onto $\mathbb{R}^{3}$. Then the set of real numbers:

$$
\omega_{i j}:=\left[u_{i-2} u_{i-1} u_{i+1} u_{i+2}\right]\left[u_{j-2} u_{j-1} u_{j+1} u_{j+2}\right]
$$

(indices in cyclic notation) defines an integer equilibrium stress on this embedding.

Theorem 2. Let $\left(p_{2}, \ldots, p_{n}\right)$ be a convex 2d drawing of a planar 3-connected graph $G_{\uparrow}$ with positive equilibrium stress $\left\{\omega_{i j}\right\}$ and designated triangular face $f_{0}=\left(p_{2} p_{3} p_{4}\right)$ embedded as the boundary face. Then we can define a cone convex embedding $\left(q_{i}\right)$ of the graph $G=\operatorname{Stack}\left(G_{\uparrow} ; v_{1} ; v_{2} v_{3} v_{4}\right)$ into $\mathbb{R}^{3}$ equipped with a positive CDV matrix $\left[M_{i j}\right]$, such that

$$
M_{i j}=\omega_{i j} \quad \text { for each internal edge }(i, j) \text { of the } 2 d \text { drawing of } G_{\uparrow}
$$

and each entry of $M$ is bounded by $O\left(n \max _{i j}\left|\omega_{i j}\right| \cdot \max _{i}\left|p_{i}\right|^{6}\right)$.

\footnotetext{
${ }^{2}$ For $2 \mathrm{~d}$ vectors $\left[p_{i} p_{j} p_{k}\right]$ is defined similarly.
} 
Proof. We can assume that $(0,0)^{T}$ lies inside the embedding of $f_{0}$. Let $\left(q_{1}, \ldots, q_{n}\right)$ be the embedding of the graph $G$, defined as follows: The embedding of $G_{\uparrow}$ is realized in the plane $\{z=1\}$ and the stacked vertex is placed at $(0,0,-1)^{T}$. The embedding is cone-convex since it describes a tetrahedron containing the origin with one face that is refined with a plane convex subdivision.

Following the structure of $G=\operatorname{Stack}\left(G_{\uparrow} ; v_{1} ; v_{2} v_{3} v_{4}\right)$, we decompose $G$ into two subgraphs: $G_{\uparrow}=G\left[\left\{v_{2}, \ldots, v_{n}\right\}\right]$ and $G_{\downarrow}:=G\left[\left\{v_{1}, v_{2}, v_{3}, v_{4}\right\}\right]$.

We first compute a CDV matrix $\left[M_{i j}^{\prime}\right]_{2 \leq i, j \leq n}$ for the embedding $\left(q_{2}, \ldots, q_{n}\right)$ of $G_{\uparrow}$. The plane embedding $\left(p_{i}\right)$ of $G_{\uparrow}$ has the equilibrium stress $\left\{\omega_{i j}\right\}_{2 \leq i, j \leq n}$. Since $\left\{q_{2}, \ldots, q_{n}\right\}$ is just a translation of $\left\{p_{2}, \ldots, p_{n}\right\}$, clearly, $\left\{\omega_{i j}\right\}_{2 \leq i, j \leq n}$ is as well an equilibrium stress for the embedding $\left(q_{2}, \ldots, q_{n}\right)$ and we can assign:

$$
M_{i j}^{\prime}:= \begin{cases}-\sum_{k \in N\left(i, G_{\uparrow}\right)} \omega_{i k} & i=j, \\ \omega_{i j} & (i, j) \in E\left(G_{\uparrow}\right), \\ 0 & \text { else. }\end{cases}
$$

Now we check the CDV equilibrium condition: for every $2 \leq i \leq n$

$$
\begin{aligned}
\sum_{2 \leq j \leq n} M_{i j}^{\prime} q_{j} & =\sum_{j \in N\left(i, G_{\uparrow}\right)} M_{i j}^{\prime} q_{j}+M_{i i}^{\prime} q_{i}=\sum_{j \in N\left(i, G_{\uparrow}\right)} M_{i j}^{\prime}\left(q_{j}-q_{i}\right)+\left(M_{i i}^{\prime}+\sum_{j \in N\left(i, G_{\uparrow}\right)} M_{i j}^{\prime}\right) q_{i} \\
& =\sum_{j \in N\left(i, G_{\uparrow}\right)} \omega_{i j}\left(q_{j}-q_{i}\right)+\left(M_{i i}^{\prime}+\sum_{j \in N\left(i, G_{\uparrow}\right)} M_{i j}^{\prime}\right) q_{i}=0 .
\end{aligned}
$$

The last transition holds since both summands equal 0 . Hence, $\left[M_{i j}^{\prime}\right]$ is a valid CDV matrix for the embedding $\left(q_{i}\right)_{2 \leq i \leq n}$ of $G_{\uparrow}$.

As a second step we compute a CDV matrix $\left[M_{i j}^{\prime \prime}\right]_{1 \leq i, j \leq 4}$ for the embedding of the tetrahedron $G_{\downarrow}$. We apply Lemma 2 for the embedding of the $K_{5}$ formed by $\left\{q_{0}=(0,0,0)^{T}, q_{1}, q_{2}, q_{3}, q_{4}\right\}$ and receive an equilibrium stress $\left\{\omega_{i j}^{\prime \prime}\right\}_{0 \leq i, j \leq 4}$. We can now derive a CDV matrix $\left[M_{i j}^{\prime \prime}\right]_{1 \leq i, j \leq 4}$ for the tetrahedron $\left\{q_{1}, q_{2}, q_{3}, q_{4}\right\}$ based on the equilibrium stresses $\left\{\omega_{i j}^{\prime \prime}\right\}_{0 \leq i, j \leq 4}$ as follows: We set

$$
M_{i j}^{\prime \prime}:= \begin{cases}-\sum_{0 \leq j \leq 4, j \neq i} \omega_{i j}^{\prime \prime}, & i=j, \\ \omega_{i j}^{\prime \prime}, & \text { otherwise }\end{cases}
$$

and see that the CDV equilibrium condition holds, by noting

$$
\begin{aligned}
\forall i \sum_{1 \leq j \leq 4} M_{i j}^{\prime \prime} q_{j} & =\sum_{1 \leq j \leq 4, j \neq i} M_{i j}^{\prime \prime} q_{j}+M_{i i}^{\prime \prime} q_{i}=\sum_{1 \leq j \leq 4, j \neq i} \omega_{i j}^{\prime \prime} q_{j}+M_{i i}^{\prime \prime} q_{i} \\
& =\sum_{0 \leq j \leq 4, j \neq i} \omega_{i j}^{\prime \prime}\left(q_{j}-q_{i}\right)-\omega_{i 0}^{\prime \prime} q_{0}+\left(\sum_{0 \leq j \leq 4, j \neq i} \omega_{i j}^{\prime \prime}+M_{i i}^{\prime \prime}\right) q_{i}=0 .
\end{aligned}
$$

The last transition holds since $\sum_{0 \leq j \leq 4, j \neq i} \omega_{i j}^{\prime \prime}\left(q_{j}-q_{i}\right)=0$ by the definition of $\left\{\omega_{i j}\right\}, q_{0}=0$, and $\sum_{0 \leq j \leq 4, j \neq i} \omega_{i j}^{\prime \prime}+M_{i i}^{\prime \prime}=0$ due to the choice of $M_{i i}^{\prime \prime}$. One can easily check that as soon as the origin lies inside the tetrahedron $\left\{q_{1}, q_{2}, q_{3}, q_{4}\right\}$ all entries $M_{i j}^{\prime \prime}$ have the same sign. We can assume that $\left[M_{i j}^{\prime \prime}\right]$ is positive, otherwise we reorder the vertices $\left\{v_{2}, v_{3}, v_{4}\right\}$. 
In the final step we extend the two CDV matrices $M^{\prime}$ and $M^{\prime \prime}$ to $G$ and combine them. Clearly, a CDV matrix padded with zeros remains a CDV matrix. Furthermore, any linear combination of CDV matrices is again a CDV matrix. Thus, we form a CDV matrix for the whole embedding $\left(q_{1}, \ldots, q_{n}\right)$ of $G$ by setting:

$$
M:=M^{\prime}+\lambda M^{\prime \prime}
$$

where $\lambda$ is a positive integer chosen such that $M$ is a positive CDV matrix. This can be done as follows.

Recall that $\left\{\omega_{i j}\right\}$ is a positive stress and $\left[M_{i j}^{\prime \prime}\right]$ is a positive CDV matrix. Hence, the only six entries in $\left[M_{i j}\right]$ that may be negative are: $M_{23}, M_{34}$ and $M_{42}$ (and their symmetric entries), for which $M_{i j}:=M_{i j}^{\prime}+\lambda M_{i j}^{\prime \prime}$ with $M_{i j}^{\prime}<0$ and $M_{i j}^{\prime \prime}>0$. Thus, we choose $\lambda$ such that $M$ is positive at these entries. To satisfy this condition we pick

$$
\lambda=\left\lfloor\max _{(i, j) \in\{(2,3),(3,4),(4,2)\}}\left(\left|M_{i j}^{\prime}\right| /\left|M_{i j}^{\prime \prime}\right|\right)\right\rfloor+1 .
$$

To bound $M_{i j}$ we notice that the entries of $M_{i j}^{\prime \prime}$ are strictly positive integers, so $\lambda=O\left(\max \left|M_{i j}^{\prime}\right|\right)$, while $\left|M_{i j}^{\prime}\right|=O\left(n \cdot \max \left|\omega_{i j}\right|\right)$ and $\left|M_{i j}^{\prime \prime}\right|=O\left(\max \left|\omega_{i j}^{\prime \prime}\right|\right)=$ $O\left(\max \left|p_{i}\right|^{6}\right)$. The bound $\left|M_{i j}\right|=O\left(n \cdot \max _{i j}\left|\omega_{i j}\right| \cdot \max _{i}\left|p_{i}\right|^{6}\right)$ follows.

\section{Realizations of Truncated Polytopes}

In this section we sum up previous results in Theorem 3 and present an embedding algorithm for truncated $3 \mathrm{~d}$ polytopes in Theorem 4. We will apply Theorem 3 also in the more general setup of Sect. 5 .

Theorem 3. Let $G=\operatorname{Stack}\left(G_{\uparrow} ; v_{1} ; v_{2} v_{3} v_{4}\right)$ and $\left(p_{2}, \ldots, p_{n}\right)$ be an integer planar embedding of $G_{\uparrow}$ with boundary face $\left(v_{2} v_{3} v_{4}\right)$ and with positive integer equilibrium stress $\left\{\omega_{i j}\right\}$. Then one can construct a grid embedding $\left(\phi_{f}\right)$ of a convex polytope with graph $G^{*}$ such that

$$
\left|\phi_{f}\right|=O\left(n^{2} \cdot \max \left|\omega_{i j}\right| \cdot \max \left|p_{i}\right|^{8}\right) \text {. }
$$

Proof. We first apply Theorem 2 to obtain a cone-convex embedding $\left(q_{1}, \ldots, q_{n}\right)$ of $G$ with a positive CDV matrix $\left[M_{i j}\right]_{1 \leq i, j \leq n}$. We then apply Lemma 1 and obtain a family of vectors $\left\{\phi_{f}\right\}_{f \in F\left(G^{*}\right)}$ fulfilling

$$
\phi_{f}-\phi_{g}=M_{i j}\left(q_{i} \times q_{j}\right), \quad \forall(f, g) \text { dual to }(i, j)-\text { edges of } G^{*} \text { and } G \text {. }
$$

Due to Theorem 1 the vectors $\left\{\phi_{f}\right\}$ form a realization of $G^{*}$ as a polytope.

To finish the proof we estimate how large the coordinates of the embedding $\left(\phi_{f}\right)$ are. To do so, let us again follow the construction of $\left(\phi_{f}\right)$ as outlined in the proof of Lemma 1. We pick one face as $f_{0} \in F(G)$, and assign $\phi_{f_{0}}=(0,0,0)^{T}$. Let us now evaluate $\phi_{f_{k}}$ for some face $f_{k} \in F(G)$. The following algebraic expression holds for all values $\left\{\phi_{f_{i}}\right\}$ :

$$
\phi_{f_{k}}=\phi_{f_{0}}+\left(\phi_{f_{1}}-\phi_{f_{0}}\right)+\ldots+\left(\phi_{f_{k-1}}-\phi_{f_{k-2}}\right)+\left(\phi_{f_{k}}-\phi_{f_{k-1}}\right) .
$$



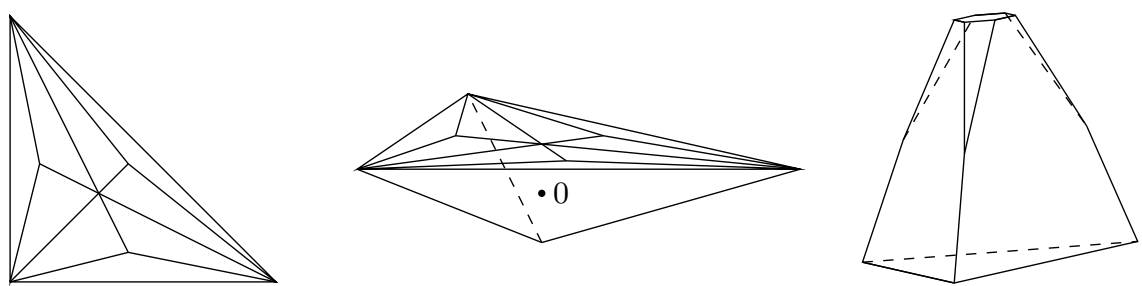

Fig. 1. A 2d embedding of $G_{\uparrow}$ (left), the cone-convex embedding of $G$ (center), and the resulting embedding of the dual (right).

Let us now consider the shortest path $f_{0}, f_{1}, \ldots, f_{k}$ in $G^{*}$ connecting the faces $f_{0}$ and $f_{k}$. Clearly, $k$ is less than $2 n-3$, and hence

$$
\begin{aligned}
\left|\phi_{f_{k}}\right| & \leq 2 n \cdot \max _{\left(f_{a}, f_{b}\right) \in E\left(G^{*}\right)}\left|\phi_{f_{a}}-\phi_{f_{b}}\right|=2 n \cdot \max _{v_{i}, v_{j} \in V(G)}\left|M_{i j}\left(q_{i} \times q_{j}\right)\right| \\
& =O\left(n \cdot\left(n \cdot \max \left|\omega_{i j}\right| \cdot \max \left|p_{i}\right|^{6}\right) \cdot \max \left|q_{i}\right|^{2}\right)=O\left(n^{2} \cdot \max \left|\omega_{i j}\right| \cdot \max \left|p_{i}\right|^{8}\right) .
\end{aligned}
$$

The bound for the entries of $M$ is due to Theorem 2 .

Next we apply Theorem 3 to construct an integer polynomial size grid embedding for truncated polytopes. To construct small integer $2 \mathrm{~d}$ embeddings with small integer equilibrium stresses we use a Lemma by Demaine and Schulz [6], which states that the graph of a stacked polytope with $n$ vertices and any distinguished face $f_{0}$ can be embedded on a $10 n^{4} \times 10 n^{4}$ grid with boundary face $f_{0}$ and with integral positive equilibrium stress $\left\{\omega_{i j}\right\}$ such that, for every edge $(i, j)$, we have $\left|\omega_{i j}\right|=O\left(n^{10}\right)$.

Theorem 4. Any truncated $3 d$ polytope with $n$ vertices can be realized with integer coordinates of size $O\left(n^{44}\right)$.

Proof. Let $G^{*}$ be the graph of the truncated polytope and $G:=\left(G^{*}\right)^{*}$ its dual. Clearly, $G$ is the graph of a stacked polytope with $(n+4) / 2$ vertices. We denote the last stacking operation (for some sequence of stacking operations producing $G)$ as the stacking of the vertex $v_{1}$ onto the face $\left(v_{2} v_{3} v_{4}\right)$ of the graph $G_{\uparrow}:=$ $G\left[V \backslash\left\{v_{1}\right\}\right]$. The graph $G_{\uparrow}$ is again a stacked graph, and hence, by the Lemma of Demaine and Schulz, there exists an embedding $\left(p_{i}\right)_{2 \leq i \leq n}$ of $G_{\uparrow}$ into $\mathbb{Z}^{2}$ with an equilibrium stress $\left\{\omega_{i j}\right\}$ satisfying the properties of Theorem 3 . We apply the theorem and obtain a polytope embedding $\left(\phi_{f}\right)$ of $G^{*}$ with bound $\left|\phi_{f}\right|=$ $O\left(n^{2} \cdot \max \left|\omega_{i j}\right| \cdot \max \left|p_{i}\right|^{8}\right)=O\left(n^{44}\right)$.

Figure 1 shows an example of our method. The computations for this example are included in the full paper.

\section{A Dual Transform for Simplicial Polytopes}

As we have seen a small grid embedding of a $3 \mathrm{~d}$ polytope can be computed when a small integer (though, not necessarily convex) embedding of its dual polytope 
with a small integral positive CDV matrix is known. However, if one wants to build a dual for an already embedded polytope, one usually does not possess such a matrix. The canonical CDV matrix associated with any embedding of a $3 \mathrm{~d}$ polytope is not helpful, since its entries, when scaled to integers, might become exponentially large. We show in this section how one can tackle this problem for a special class of polytopes. In particular, we require that the original polytope is simplicial, it contains a vertex of degree 3, and its maximum vertex degree is bounded.

Before proceeding, let us review how the canonical stress associated with an orthogonal projection of a $3 \mathrm{~d}$ polytope in the $\{z=0\}$ plane can be described. The assignment of heights to the interior vertices of a $2 \mathrm{~d}$ embedding resulting in a polyhedral surface is called a (polyhedral) lifting. By the Maxwell-Cremona correspondence the equilibrium stresses of a $2 \mathrm{~d}$ embedding of a planar 3 -connected graph and its liftings are in 1-1 correspondence. Moreover, the bijection between liftings and stresses can be defined as follows. Let $\left(p_{i}\right)$ be a $2 \mathrm{~d}$ drawing of a triangulation and let $\left(q_{i}\right)$ be the $3 \mathrm{~d}$ embedding induced by some lifting. We map this lifting to the equilibrium stress $\left\{\omega_{i j}\right\}$ by assigning to every edge $(i, j)$ separating the faces $\left(v_{i} v_{j} v_{k}\right)$ (on the left) and $\left(v_{i} v_{j} v_{l}\right)$ (on the right)

$$
\omega_{i j}:=\frac{\left[q_{i} q_{j} q_{k} q_{l}\right]}{\left[p_{i} p_{j} p_{k}\right]\left[p_{l} p_{j} p_{i}\right]}
$$

This mapping is a bijection between the space of liftings and the space of equilibrium stresses. The expression (3) is a slight reformulation of the form presented in Hopcroft and Kahn [8, Equation 11].

We continue by studying the spaces of equilibrium stresses for triangulations. A graph formed by a cycle $v_{1}, \ldots, v_{n}$ with an additional vertex $v_{0}$, called center, that is adjacent to every other vertex, is called a wheel; we denote it as $\mathrm{W}\left(v_{0} ; v_{1} \ldots v_{n}\right)$. A wheel that is a subgraph of a triangulation $G$ with $v_{i} \in V(G)$ as center is denoted by $W_{i}$. Every triangulation can be "covered" with a set of wheels $\left\{W_{i}\right\}_{v_{i} \in V(G)}$, such that every edge is covered four times.

Lemma 3. Let $\left(p_{0}, \ldots, p_{n}\right)$ be an embedding of a wheel $\mathrm{W}\left(v_{0} ; v_{1} \ldots v_{n}\right)$ in $\mathbb{R}^{2}$. Then the following expression defines an equilibrium stress:

$$
\omega_{i j}= \begin{cases}-1 /\left[p_{i} p_{i+1} p_{0}\right] & j=i+1,1 \leq i \leq n, \\ {\left[p_{i-1} p_{i} p_{i+1}\right] /\left(\left[p_{i-1} p_{i} p_{0}\right]\left[p_{i} p_{i+1} p_{0}\right]\right)} & j=0,1 \leq i \leq n\end{cases}
$$

The equilibrium stress for the embedding $\left(p_{i}\right)$ is unique up to a renormalization.

Proof. This stress coincides with (3) from the lifting of $W$ with $z_{0}=1$ and $z_{i}=0$ for $1 \leq i \leq n$ and so is an equilibrium stress. The space of the stresses is 1-dimensional, since the space of the polyhedral liftings is 1-dimensional.

Definition 4. 1. For a wheel $W$ embedded in the plane we refer to the equilibrium stress defined in Lemma 3 as its small atomic stress and denote it as $\left\{\omega_{i j}^{a}(W)\right\}$. 
2. We call the stress $\left\{\Omega_{i j}^{a}(W)\right\}$ that is obtained by the renormalization of $\left\{\omega_{i j}^{a}(W)\right\}$ by the factor $\prod_{1 \leq j \leq n}\left[p_{j} p_{j+1} p_{0}\right]$, the large atomic stress of $W$.

We point out that the large atomic stresses are products of $\operatorname{deg}\left(v_{0}\right)-1$ triangle areas multiplied by 2 , and so, $\left\{\Omega_{i j}^{a}(W)\right\}$ is a set of integers if $W$ is realized with integer coordinates.

Theorem 5 (Wheel-decomposition). Let $G$ be a triangulation. Every equilibrium stress $\left\{\omega_{i j}\right\}$ of an embedding $\left(p_{1}, \ldots, p_{n}\right)$ of $G$ can be expressed as a linear combination of the small atomic stresses on the wheels $\left\{W_{i}\right\}$ :

$$
\omega=\sum_{i \leq n} \alpha_{i} \omega^{a}\left(W_{i}\right)
$$

where the coefficients $\alpha_{i}$ are the heights (i.e., z-coordinates) of the corresponding vertices $v_{i}$ in the Maxwell-Cremona lifting of $\left(p_{1}, \ldots, p_{n}\right)$ induced by $\left\{\omega_{i j}\right\}$.

Proof. Let $\left(q_{1}, \ldots, q_{n}\right)$ be the Maxwell-Cremona lifting of $\left(p_{i}\right)$ by means of the stress $\left\{\omega_{i j}\right\}$. We rewrite this stress (given by Equation 3) using

$$
\left[q_{1} q_{2} q_{3} q_{4}\right]=\sum_{1 \leq i \leq 4}(-1)^{i+1} z_{i}\left[p_{i+1} p_{i+2} p_{i+3}\right]
$$

(with cyclic notation for indices) and obtain

$$
\omega_{i j}=z_{i} \frac{\left[p_{j} p_{k} p_{l}\right]}{\left[p_{i} p_{j} p_{k}\right]\left[p_{l} p_{j} p_{i}\right]}+z_{j} \frac{\left[p_{l} p_{k} p_{i}\right]}{\left[p_{i} p_{j} p_{k}\right]\left[p_{l} p_{j} p_{i}\right]}-z_{k} \frac{1}{\left[p_{i} p_{j} p_{k}\right]}-z_{l} \frac{1}{\left[p_{l} p_{j} p_{i}\right]},
$$

which is exactly the decomposition of $\omega_{i j}$ into small atomic wheel stresses.

Theorem 6. Let $\left(q_{1}, \ldots, q_{n}\right)$ be an embedding of a triangulation $G$ into $\mathbb{Z}^{3}$, whose projection $\left(p_{1}, \ldots, p_{n}\right)$ to the plane $\{z=0\}$ is a noncrossing embedding of $G$ with boundary face $\left(v_{1} v_{2} v_{3}\right)$. Then one can construct a positive integer equilibrium stress $\left\{\omega_{i j}\right\}$ for the embedding $\left(p_{1}, \ldots, p_{n}\right)$ such that

$$
\left|\omega_{i j}\right|<\left(\max _{i \leq n}\left|q_{i}\right|\right)^{2 \Delta_{\mathrm{G}}+5} .
$$

Proof. We start with the equilibrium stress $\left\{\widetilde{\omega}_{i j}\right\}$ as specified by (3) for the embedding $\left(p_{i}\right)$. Since all the coordinates are integers, all stresses are bounded by

$$
\frac{1}{\mathrm{~L}^{4}} \leq \frac{1}{\left|\left[p_{i} p_{j} p_{k}\right]\right|\left|\left[p_{l} p_{j} p_{i}\right]\right|} \leq\left|\widetilde{\omega}_{i j}\right| \leq\left|\left[q_{i} q_{j} q_{k} q_{l}\right]\right| \leq \mathrm{L}^{3},
$$

for $\mathrm{L}=2 \max _{i \leq n}\left|q_{i}\right|$.

We are left with making these stresses integral while preserving a polynomial bound. The stress $\left\{\widetilde{\omega}_{i j}\right\}$ can be written as a linear combination of large atomic stresses of the wheels $W_{i}$ by means of the Wheel-decomposition Theorem,

$$
\widetilde{\omega}_{i j}=\alpha_{i} \Omega_{i j}^{a}\left(W_{i}\right)+\alpha_{j} \Omega_{i j}^{a}\left(W_{j}\right)+\alpha_{k} \Omega_{i j}^{a}\left(W_{k}\right)+\alpha_{l} \Omega_{i j}^{a}\left(W_{l}\right) .
$$


Since all points $p_{i}$ have integer coordinates, the large atomic stresses are integers as well. Moreover, each of them, as a product of $\operatorname{deg}\left(v_{k}\right)-1$ triangle areas, is bounded by $\left|\Omega_{i j}^{a}\left(W_{k}\right)\right| \leq \mathrm{L}^{2\left(\Delta_{\mathrm{G}}-1\right)}$.

To make the $\widetilde{\omega}_{i j}$ s integral we round the coefficients $\alpha_{i}$ down. To guarantee that the rounding does not alter the signs of the stress, we scale the atomic stresses (before rounding) with the factor

$$
C=4 \max _{i, j, k}\left|\Omega_{i j}^{a}\left(W_{k}\right)\right| / \min _{i, j}\left|\widetilde{\omega}_{i j}\right|
$$

and define as the new stress:

$$
\omega_{i j}:=\left\lfloor C \alpha_{i}\right\rfloor \Omega_{i j}^{a}\left(W_{i}\right)+\left\lfloor C \alpha_{j}\right\rfloor \Omega_{i j}^{a}\left(W_{j}\right)+\left\lfloor C \alpha_{k}\right\rfloor \Omega_{i j}^{a}\left(W_{k}\right)+\left\lfloor C \alpha_{l}\right\rfloor \Omega_{i j}^{a}\left(W_{l}\right) .
$$

Clearly,

$$
\begin{aligned}
\left|\omega_{i j}-C \widetilde{\omega}_{i j}\right| & =\left|\sum_{\tau=i, j, k, l}\left(\left\lfloor C \alpha_{\tau}\right\rfloor-C \alpha_{\tau}\right) \Omega_{i j}^{a}\left(W_{\tau}\right)\right| \\
& \leq \sum_{\tau=i, j, k, l}\left|\Omega_{i j}^{a}\left(W_{\tau}\right)\right| \leq 4 \max _{i, j, k}\left|\Omega_{i j}^{a}\left(W_{k}\right)\right|=C \min _{i, j}\left|\widetilde{\omega}_{i j}\right| \leq C\left|\widetilde{\omega}_{i j}\right|
\end{aligned}
$$

and $\operatorname{so} \operatorname{sign}\left(\omega_{i j}\right)=\operatorname{sign}\left(C \widetilde{\omega}_{i j}\right)=\operatorname{sign}\left(\widetilde{\omega}_{i j}\right)$.

Therefore, the constructed equilibrium stress $\left\{\omega_{i j}\right\}$ is integral and positive. We conclude the proof with an upper bound on its size. Since $C<4 \mathrm{~L}^{2\left(\Delta_{\mathrm{G}}-1\right)} \mathrm{L}^{4}$,

$$
\begin{aligned}
\left|\omega_{i j}\right| & \leq\left|\sum_{\tau=i, j, k, l}\left(C \alpha_{\tau} \pm 1\right) \Omega_{i j}^{a}\left(W_{\tau}\right)\right| \leq C\left|\widetilde{\omega}_{i j}\right|+\sum_{\tau=i, j, k, l}\left|\Omega_{i j}^{a}\left(W_{\tau}\right)\right| \\
& \leq C \max \left|\widetilde{\omega}_{i j}\right|+4 \max \left|\Omega_{i j}^{a}\left(W_{k}\right)\right| \leq 4 \mathrm{~L}^{2 \Delta_{\mathrm{G}}+2} \cdot \mathrm{L}^{3}+4 \mathrm{~L}^{2 \Delta_{\mathrm{G}}-2}=O\left(\mathrm{~L}^{2 \Delta_{\mathrm{G}}+5}\right) .
\end{aligned}
$$

Combining Theorem [ 6 and Theorem 3 leads to the following result:

Theorem 7. Let $\left(q_{2}, \ldots, q_{n}\right)$ be an integer embedding of a simplicial 3d polytope with graph $G_{\uparrow}$, such that the orthogonal projection into the plane $\{z=0\}$ gives a planar $2 d$ embedding $\left(p_{2}, \ldots, p_{n}\right)$ with boundary face $\left(v_{2} v_{3} v_{4}\right)$. Then we can construct an embedding $\left(\phi_{f}\right)_{f \in F(G)}$ of a graph dual to $G=\operatorname{Stack}\left(G_{\uparrow} ; v_{1} ; v_{2} v_{3} v_{4}\right)$ with integer coordinates bounded by

$$
\left|\phi_{f}\right|=O\left(n^{2} \max \left|q_{i}\right|^{2 \Delta_{\mathrm{G}}+13}\right) .
$$

We remark that the algorithms following the lifting approach generate embeddings that fulfill the conditions of the above theorem. Using a more technical analysis we can even show that the following stronger version of Theorem 7 holds. The proof of the theorem can be found in the full version of the paper.

Theorem 8. Let $G$ be a triangulation with at least one vertex of degree 3, and let $\left(q_{i}\right)$ be an integer realization of $G$ as a convex polytope. Then there is a realization $\left(\phi_{f}\right)_{f \in F(G)}$ of the dual graph $G^{*}$ as a convex polytope with integer coordinates bounded by

$$
\left|\phi_{f}\right|<\max \left|q_{i}\right|^{O\left(\Delta_{\mathrm{G}}\right)} .
$$




\section{References}

1. Andrews, G.E.: A lower bound for the volume of strictly convex bodies with many boundary lattice points. Trans. Amer. Math. Soc. 99, 272-277 (1961)

2. Bárány, I., Rote, G.: Strictly convex drawings of planar graphs. Documenta Math. 11, 369-391 (2006)

3. Buchin, K., Schulz, A.: On the number of spanning trees a planar graph can have. In: de Berg, M., Meyer, U. (eds.) ESA 2010, Part I. LNCS, vol. 6346, pp. 110-121. Springer, Heidelberg (2010)

4. Das, G., Goodrich, M.T.: On the complexity of optimization problems for 3-dimensional convex polyhedra and decision trees. Computational Geometry: Theory and Applications 8(3), 123-137 (1997)

5. de Fraysseix, H., Pach, J., Pollack, R.: How to draw a planar graph on a grid. Combinatorica 10(1), 41-51 (1990)

6. Demaine, E.D., Schulz, A.: Embedding stacked polytopes on a polynomial-size grid. In: Proc. 22nd ACM-SIAM Symposium on Discrete Algorithms (SODA), pp. 1177-1187. ACM Press (2011)

7. Eades, P., Garvan, P.: Drawing stressed planar graphs in three dimensions. In: Brandenburg, F.J. (ed.) GD 1995. LNCS, vol. 1027, pp. 212-223. Springer, Heidelberg (1996)

8. Hopcroft, J.E., Kahn, P.J.: A paradigm for robust geometric algorithms. Algorithmica 7(4), 339-380 (1992)

9. Lovász, L.: Steinitz representations of polyhedra and the Colin de Verdière number. J. Comb. Theory, Ser. B 82(2), 223-236 (2001)

10. Maxwell, J.C.: On reciprocal figures and diagrams of forces. Phil. Mag. Ser. 27, 250-261 (1864)

11. Mor, A.R., Rote, G., Schulz, A.: Small grid embeddings of 3-polytopes. Discrete \& Computational Geometry 45(1), 65-87 (2011)

12. Onn, S., Sturmfels, B.: A quantitative Steinitz' theorem. Beiträge zur Algebra und Geometrie 35, 125-129 (1994)

13. Richter-Gebert, J.: Realization Spaces of Polytopes. Lecture Notes in Mathematics, vol. 1643. Springer (1996)

14. Rote, G., Santos, F., Streinu, I.: Expansive motions and the polytope of pointed pseudo-triangulations. Discrete and Computational Geometry-The GoodmanPollack Festschrift 25, 699-736 (2003)

15. Schulz, A.: Drawing 3-polytopes with good vertex resolution. Journal of Graph Algorithms and Applications 15(1), 33-52 (2011)

16. Steinitz, E.: Polyeder und Raumeinteilungen. In: Encyclopädie der mathematischen Wissenschaften, vol. 3-1-2 (Geometrie), ch. 12, pp. 1-139. B.G. Teubner, Leipzig (1916)

17. Whitney, H.: Congruent graphs and the connectivity of graphs. Amer. J. Math. 54, 150-168 (1932) 